An electrooptical muscle contraction sensor

\author{
Alessio Chianura and Mario E Giardini \\ Medical and Biological Engineering and Computing \\ $48(2010) \quad 731-734$
}

This is an author-generated version

The final publication is available at www.springerlink.com DOI : $10.1007 / \mathrm{s} 11517-010-0626-\mathrm{x}$ 


\title{
An electrooptical muscle contraction sensor
}

\author{
Alessio Chianura ${ }^{*}$ and Mario E. Giardini ${ }^{\#}$ \\ *Università degli Studi di Pavia - Dipartimento di Elettronica - Pavia - Italy \\ ${ }^{\#}$ Istituto Nazionale per la Fisica della Materia - INFM - Genova - Italy
}

\#Corresponding author:

\author{
current affiliation and present address: \\ Mario E. Giardini \\ University of St Andrews \\ SUPA - School of Physics and Astronomy \\ J.F. Allen Physics Research Laboratories \\ North Haugh \\ St Andrews KY16 9SS \\ United Kingdom \\ E-mail: mario.giardini@st-andrews.ac.uk \\ Tel. +44 133446.3439 \\ Fax +44 133446.3104
}

The total number of words of the manuscript, including entire text from title page to figure legends: 1522

The number of words of the abstract: $\mathbf{8 8}$

The number of figures: 3

(The number of tables: 0 ) 


\section{ABSTRACT}

An electrooptical sensor for the detection of muscle contraction is described. Infrared light is injected into the muscle, the backscattering is observed, and the contraction is detected by measuring the change, that occurs during muscle contraction, between the light scattered in the direction parallel and perpendicular to the muscle cells. With respect to electromyography and to optical absorption-based sensors, our device has the advantage of lower invasiveness, of lower sensitivity to electromagnetic noise and to movement artifacts, and of being able to distinguish between isometric and isotonic contractions.

\section{KEYWORDS}

muscle contraction, optical device, infrared, isometric, isotonic 


\section{Introduction}

In the design of an active prosthetic device, the detection of a signal suitable as a trigger or as a proportional controller for the prosthesis actuation is among the key issues. In current clinical applications, surface electromyography (EMG), that detects the electric signals that underlie muscle contraction, is in widespread use $\mathrm{e}^{1,2,3}$. The patient is trained to contract appropriate muscles when he needs to actuate a specific movement in the prosthesis.

However EMG, based on the detection of low voltage signals at high impedance through electrodes applied on the surface of the skin, it is highly sensitive to electromagnetic interference ${ }^{4}$. Moreover, in order to reduce the impedance and to minimize movement artifacts, electrodes are put in direct contact with the tissue, often with a significant pressure, and the consequent tissue reaction and eventual reduction in tissue blood flow may cause malfunction and/or tissue damage , $^{5,6}$.

For these reasons, in order to detect muscle contraction, optical techniques are particularly appealing, as they are intrinsically free from electromagnetic noise and, in principle, are not invasive on the tissues. As the near-infrared optical absorption of muscle is dominated by blood ${ }^{7}$ and as, during contraction, the muscle undergoes blood depletion, it has been demonstrated that the contraction can be detected as a decrease in the optical absorption of the muscle ${ }^{8,9}$. Though this technique can indeed be used to generate a signal for prosthesis actuation, the sensitivity is low and, so far, viable sensor configurations have shown to be prone to artifacts due to patient movement ${ }^{10}$.

\section{Materials and methods}

Our solution for the optical detection of muscle contraction is based on the observation that muscle cells are shaped as elongated fibers, aligned along the main muscle axis. In muscle tissue, we can therefore expect light to be scattered anisotropically. Namely, if we inject light into the muscle using a suitable point light source applied to the overlaying skin, the backscattered light, collected through the skin a few centimeters away from the source, depends on the collection point position with respect to the muscle fiber direction ${ }^{11}$. In particular, the light scattered in a direction perpendicular to the muscle fibers differs from the light scattered parallel to the fibers. As the muscle contracts, the fiber aspect ratio changes, and the scattering anisotropy varies accordingly. We have therefore designed our sensor to detect such variation, responding to contraction with intrinsic rejection to nonanisotropic signals, such as those resulting from patient movement.

The sensor head is constituted of a round PVC housing, $50 \mathrm{~mm}$ in diameter and $8 \mathrm{~mm}$ thick, with five $5 \mathrm{~mm}$ PMMA windows arranged on the vertices and in the center of an ideal square with 40 $\mathrm{mm}$ diagonals. Such housing contains four photodiodes (BPW34, Siemens), arranged behind the peripheral windows, and a LED emitting at $880 \mathrm{~nm}$ (HIRL 8810, Rodan) behind the central one. 
Using such configuration, the sensor collects light coming from a depth on the order of $2 \mathrm{~cm}$ under the skin surface ${ }^{7}$.

A $1 \mathrm{~m}$ multicore cable connects the photodiodes and the LED to an appropriate photodiode preamplifier and a continuous-wave LED driver.

Inside the head, the cable is separated in its individual cores, that run in channels, held in place by an optically absorbing potting compound. This minimizes the optical crosstalk between each component. Figure 1 shows the lower part of the housing, with the channels, rendered from its CAD design (a), and a photograph of the assembled sensor head (b).

The sensor is placed directly on the skin, over a muscle. The LED emits light through the skin and into the muscle. It is oriented so that two photodiodes (1 and 3) collect the light scattered in the direction of the muscle fibers, while the other two photodiodes ( 2 and 4 ) collect the light scattered perpendicularly to such direction (photocurrents $\mathrm{I}_{/ /}$and $\mathrm{I}_{\perp}$ respectively).

$I_{/ /}$and $I_{\perp}$ are time-multiplexed, with opposite signs, on the input node of a current integrator. A feedback loop controls the duty cycle of the multiplexer balancing the output of the integrator for zero signal at steady state. Any imbalance between $I_{/ /}$and $I_{\perp}$ faster than the loop time constant (22 s) appears as a non-zero signal on the integrator output. A simplified preamplifier schematic diagram is reported in Figure $2 b$.

\section{Results and discussion}

The sensor has been tested on a 28 year old male volunteer, placed over his biceps muscle, held in place by an elastic dark cloth bandage, taking care not to occlude blood circulation. The volunteer has then performed series of isotonic contractions (i.e., at constant muscle force), lifting a 8 $\mathrm{kg}$ dumbbell, and series of isometric contractions (i.e., at constant muscle length) against a fixed obstacle. Typical signals detected at the integrator output are reported in Figure 3.

Stable and consistent individual contraction signals can be easily distinguished against the steady-state baseline, and the shape of the signals corresponding to isotonic and isometric contractions is clearly different. In particular, in a first phase of the isotonic contractions, the muscle is rapidly depleted of blood, and then the differential optical scattering contribution due to the shortening of the muscular fibers prevails. Conversely, in isometric contractions, the muscle fibers are constrained to a quasi-constant length. Therefore, differential scattering contributes to a first phase of the signal only, and then haematic depletion dominates. As, in our configuration, differential scattering and blood depletion contribute to the signal with opposing signs, the isometric and isotonic signals present opposing leading and trailing edge slopes and steady-state values. 


\section{Conclusions}

For the quality of the signals obtained, the device appears promising as an alternative to surface EMG for monitoring muscle contraction. As described, it enables a clear non-invasive detection of the contraction signal, with the substantial advantages on electromagnetic noise that optical methods present over low-signal electric detection, and with the additional capability to distinguish between isometric and isotonic contractions.

\section{References}

1 Merletti R, Parker PA (eds) (2004), Electromyography: Physiology, Engineering and Noninvasive Applications, Wiley-IEEE, USA

2 Reaz MBI, Hussain MS, Mohd-Yasin F (2006) Techniques of EMG signal analysis: detection, processing, classification and applications. Biol. Proced. Online 8: 11-35

${ }^{3}$ Parker P, Englehart K, Hudgins B (2006) Myoelectric signal processing for control of powered limb prostheses. J Electromyogr. Kinesiol. 16: 541-548

${ }^{4}$ Andreasen LNS, Struijk UJJ (2003) Artefact reduction with alternative cuff configurations. IEEE Trans. Biomed. Eng 50: 1160-1166

${ }^{5}$ Lai JCK, Schoen MP, Gracia AP, Naidu DS, Leung SW (2007) Prosthetic devices: challenges and implications of robotic implants and biological interfaces. Proc. Institut. Mech. Eng. H - J. Eng. Med. 221: 173-183

${ }^{6}$ Sood A, Taylor JS, Billock JN (2003) Contact Dermatitis to a limb prosthesis. Am. J. Contact Dermatitis 14: $169-171$

${ }^{7}$ Tuchin V (2000) Tissue Optics, SPIE Publishing, USA

${ }^{8}$ Gelmetti A, Giardini ME, Lago P, Pavesi R, Zambarbieri D, Maestri R, Felicetti G (1997) Preliminary Study of Muscle Contraction Assessment by NIR Spectroscopy. SPIE Proc. 3199: 61-67

${ }^{9}$ Bianchi T, Zambarbieri D, Beltrami G, Verni G (1999) NIRS monitoring of muscle contraction to control a prosthetic device. SPIE Proc. 3570: 157-163 
${ }^{10}$ Lopez NM, di Sciascio F, Soria CM, Valentinuzzi ME (2009) Robust EMG sensing system based on data fusion for myoelectric control of a robotic arm. Biomed Eng Online 8: Art. No. 5

${ }^{11}$ Fomenko VN, Shwarts FM, Shwarts MA (1998) Exact description of the photon migration in anisotropically scattering media. SPIE Proc. 3566: 47-56 


\section{Figure legends}

FIG. 1. CAD rendering of the sensor head housing (a) and photograph of the assembled sensor head (b)

FIG. 2. Simplified schematic diagram of the sensor amplifier. (a): photodiodes D1 and D3 collect the light scattered parallel to the muscle fibers, D2 and D4 the light perpendicular to the fibers. (b): the photocurrents are integrated on $\mathrm{C}_{\mathrm{i}}$ to yield the output signal $\mathrm{V}_{\mathrm{s}}$. The background, filtered with time constant $\mathrm{R}_{\mathrm{f}} \mathrm{C}_{\mathrm{f}}$ is compared with a triangular signal $\mathrm{V}_{\mathrm{t}}$ to balance the photocurrents.

FIG. 3. Sensor output for isotonic (a) and isometric (b) muscle contractions. 


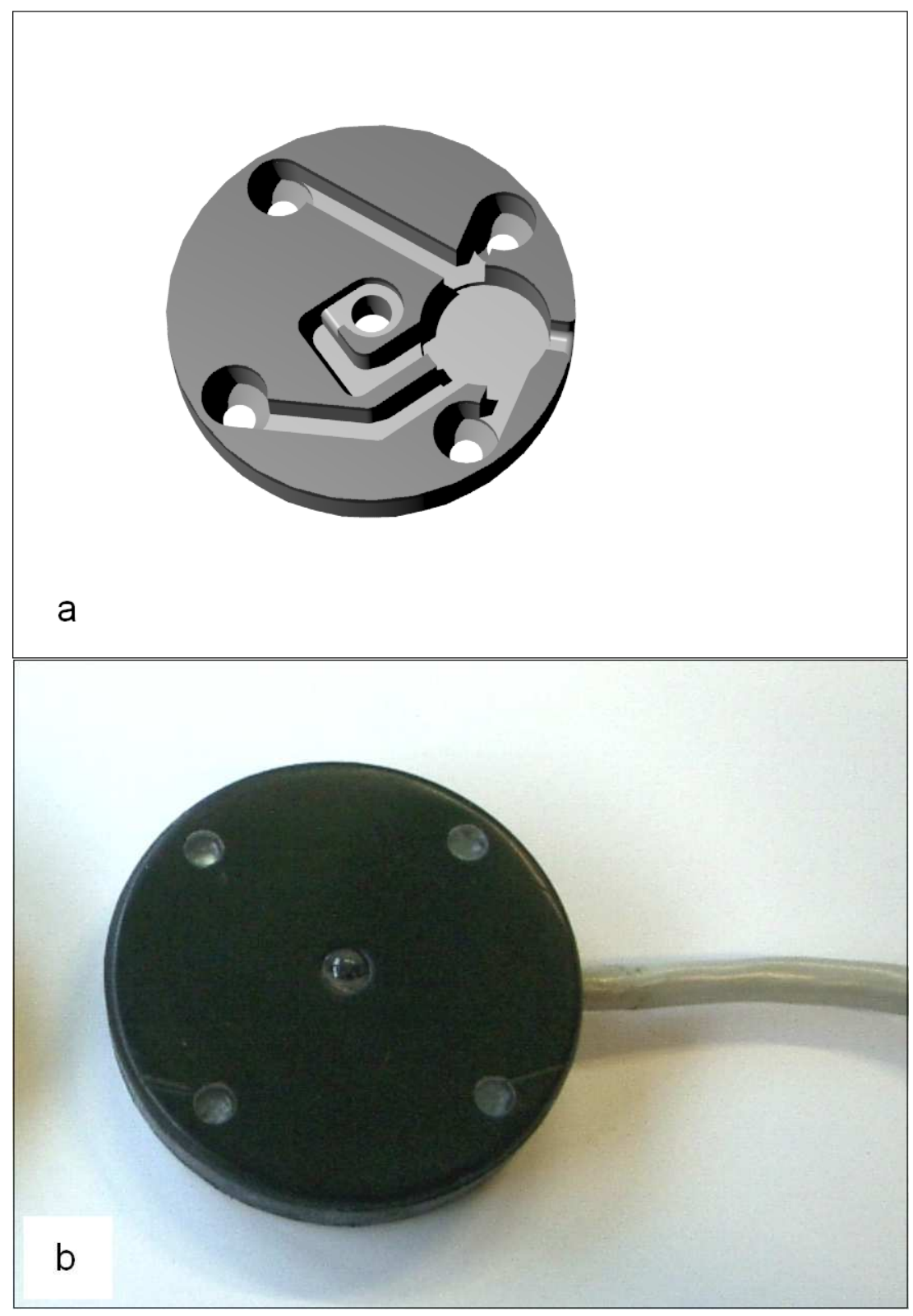

Figure 1 
$a$
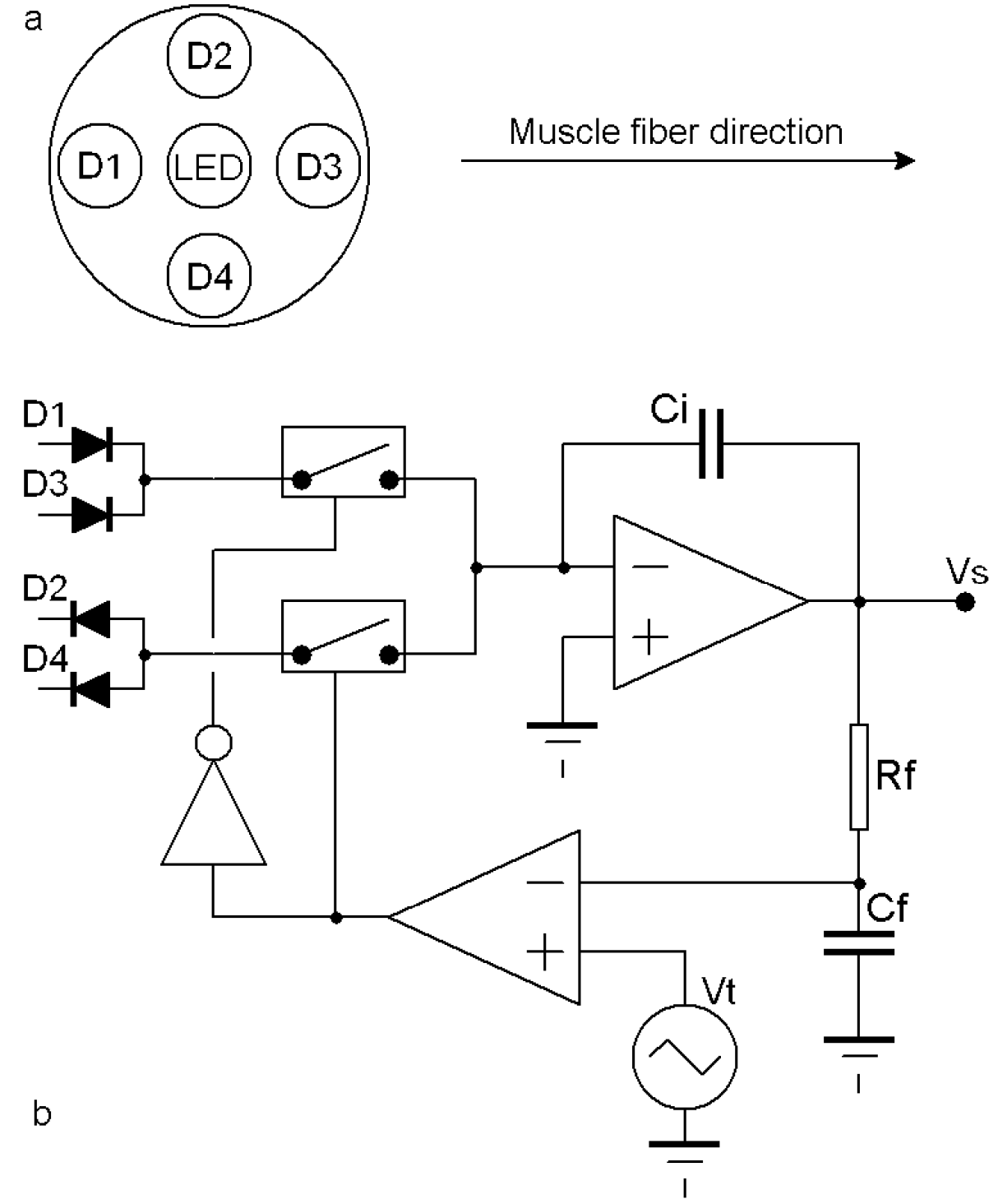

Figure 2 

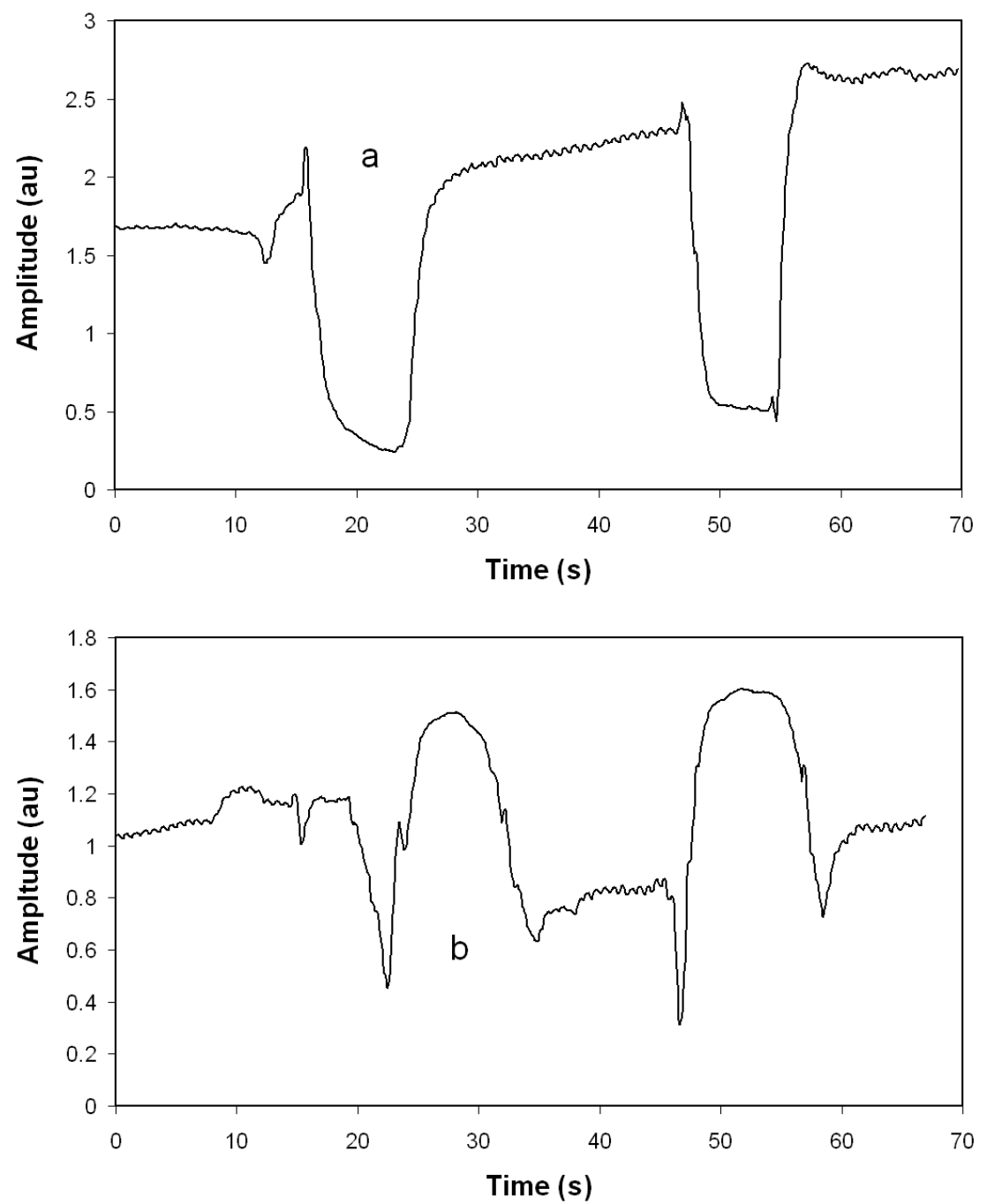

Figure 3 